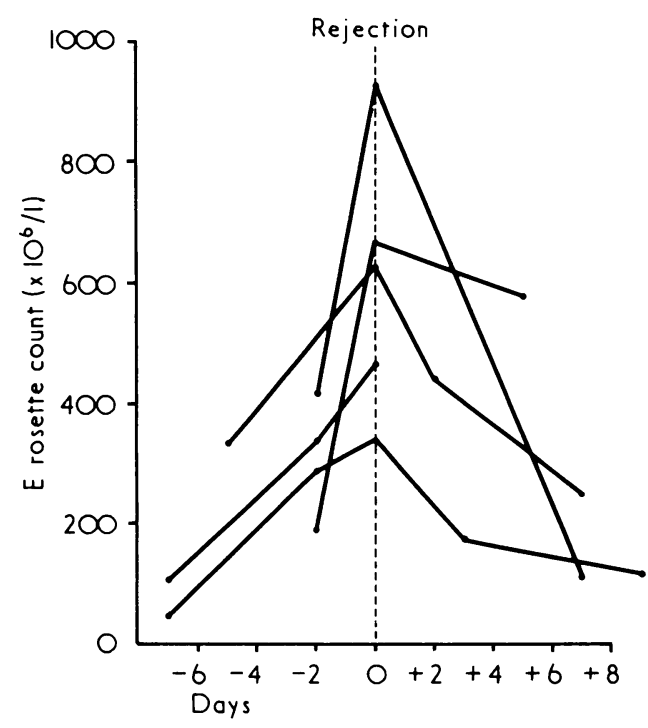

FIG 3-Rejection crises with increased E rosette counts. Steroid treatment begun on day 0 .

circulating E rosettes to steroids seen in these allograft recipients compared with the high sensitivity of $\mathrm{E}$ rosettes to steroids in normal people 2 may be related to the graft itself, subclinical rejection possibly leading to an abnormally high $\mathrm{T}$ cell level. Possibly the association of azathioprine with steroids modifies steroid sensitivity of $\mathrm{T}$ cells in selecting a steroid-resistant $\mathrm{T}$ cell subset.

The somewhat paradoxical results concerning rejection crises are still more difficult to interpret. One might have thought that immunosuppressive treatment would have produced a $\mathrm{T}$ cell depression and that rejection would occur when this depression became insufficient. We, however, found that maintenance of immunosuppression only slightly altered the $\mathrm{E}$ rosette counts and that they could not be used as a reliable measure of the efficacy of treatment. More paradoxical was that most crises were associated with a rapid decrease in $\mathrm{E}$ rosettes before steroid treatment and that clinically successful steroid pulses, which decrease the number of $\mathrm{E}$ rosettes in normal people, ${ }^{2}$ induced in these patients an increase in $\mathrm{E}$ rosettes. This phenomenon may be specific for rejection, since it was observed in only two cases in the absence of clinical evidence of rejection.

One may wonder whether in such cases there was merely a loss of the marker expression under steroid influence or whether $T$ cells were indeed depleted at the onset of rejection and replaced by another cell population. In the latter hypothesis the loss of $\mathrm{T}$ cells might eventually be the result of a suppressor $T$ cell population having a favourable action on graft survival, acting either on $B$ cells or on other $T$ cells including those involved in cytolytic processes. ${ }^{3}$ The nature of the cells replacing the $\mathrm{E}$ rosette forming cells, however, remains unknown. Preliminary results in our laboratory using B cell evaluations by the EAC rosette technique 4 show that decreases in $\mathrm{E}$ rosette counts during rejection are not associated with an increase in B cells but are associated with a large excess of "null" cells, as defined by the absence of the two markers used here, which include the newly defined " $K$ " cells. ${ }^{5}$ Interestingly, $\mathrm{K}$ cells are highly sensitive to steroids. ${ }^{6}$

Finally, our results remain impossible to explain in relation to current concepts of the mechanisms of organ transplant rejection. Nevertheless, they show the need for caution in judging the efficacy of immunosuppressive treatment in allograft recipients on the basis of available $B$ and $T$ cell markers. Thus one cannot use $\mathrm{E}$ rosettes for monitoring anti-rejection treatment except for the detection of the most severe states of immunosuppression, which may expose the patient to severe infections, as indicated by the persistent low $\mathrm{E}$ rosette counts in patients with lethal infections.

\section{References}

${ }^{1}$ Bach, J F, Transplantation Reviews, 1973, 16, 196.

2 Bach, J F, et al, Transplantation Proceedings, 1975, 7, 25.

${ }^{3}$ Gershon, R K, Contemporary Topics in Immunobiology, 1974, 3, 1.

4 Nussenzweig, V, and Pincus, C S, Contemporary Topics in Immunobiology, $1972,1,69$.

${ }^{5}$ MacLennan, M, Connell, G E, and Gotch, F M, Immunology, 1974, 26, 303.

${ }^{6}$ Descamps, B, Gagnon, R, and Crosnier, J, Transplantation Proceedings, In press.

\title{
Response and survival in advanced breast cancer after two non-cross-resistant combinations
}

\author{
C BRAMBILLA, M DE LENA, A ROSSI， P VALAGUSSA， G BONADONNA
}

British Medical fournal, 1976, 1, 801-804

\section{Summary}

A prospective study with two cytotoxic combinations (cyclophosphamide, methotrexate, and fluorouracil (CMF), and adriamycin plus vincristine (AV)) was carried out in 110 patients with advanced breast cancer. There was

\footnotetext{
Division of Clinical Oncology F, Istituto Nazionale Tumori, Milan, Italy

C BRAMBILLA, MD, clinical assistant

M DE LENA, MD, clinical assistant

A ROSSI, MD, research fellow

P VALAGUSSA, BS, consultant statistician

G BONADONNA, MD, associate director
}

no significant difference between the treatment groups in the response rate after primary treatment, the median duration of response, and the median survival. In both groups responders survived for longer than non-responders. Secondary treatment after crossover for progression or relapse resulted in response rates of $35 \%$ for $\mathbf{A V}$ and $20 \%$ for CMF. Toxicity was mainly represented by reversible haemosuppression. These results are comparable with those obtained with other multiple-drug regimens, and combination chemotherapy alone seems to have reached a plateau in its capacity to control disseminated breast cancer.

\section{Introduction}

The control of advanced breast cancer at any stage of the disease presents many challenging problems. The advent of cyclical 
combination chemotherapy has increased greatly the proportion of responding patients with clinically disseminated disease. Many reports have confirmed that different multiple-drug regimens can produce complete or partial remissions in $50-87 \%$ of patients. ${ }^{1-4}$ Furthermore, there is preliminary evidence that the response rate is probably increased by adding adriamycin to some regimens. ${ }^{45}$

In all but two studies the response rate induced by multipledrug regimens has been greater than that induced by single agents. Ahman et $a l^{6}$ and Gottlieb et al, ${ }^{7}$ however, reported that the percentage of complete and partial remissions obtained with adriamycin were comparable to those produced by triple and quintuple drug combinations.

The impact of higher remission rates on survival is currently under investigation. The treatment of choice is not yet clearly identifiable, and neither the length of complete response or survival after primary treatment indicate that there is a consistent fraction of patients who can be maintained free of disease for a long period. For these reasons, we began study in 1973 to test the therapeutic effects of two independent drug combinations. Our intention was to develop effective non-crossresistant regimens to be used after primary treatment failure and possibly in sequence in future trials. The preliminary results in the first series of 80 patients have been published. ${ }^{2}$ We report here the final evaluation, which includes data on the duration of response and survival.

\section{Patients and methods}

The treatment and dose schedules are shown in fig 1 . Before randomisation patients were stratified according to menopausal status (menopause had occurred less than 1 year, 1-5 years, 6-10 years, or more than 10 years earlier), disease free interval (simultaneous with treatment, 1-5 years long, or longer than 5 years), and site of dominant lesion (soft tissue, viscera, bone).

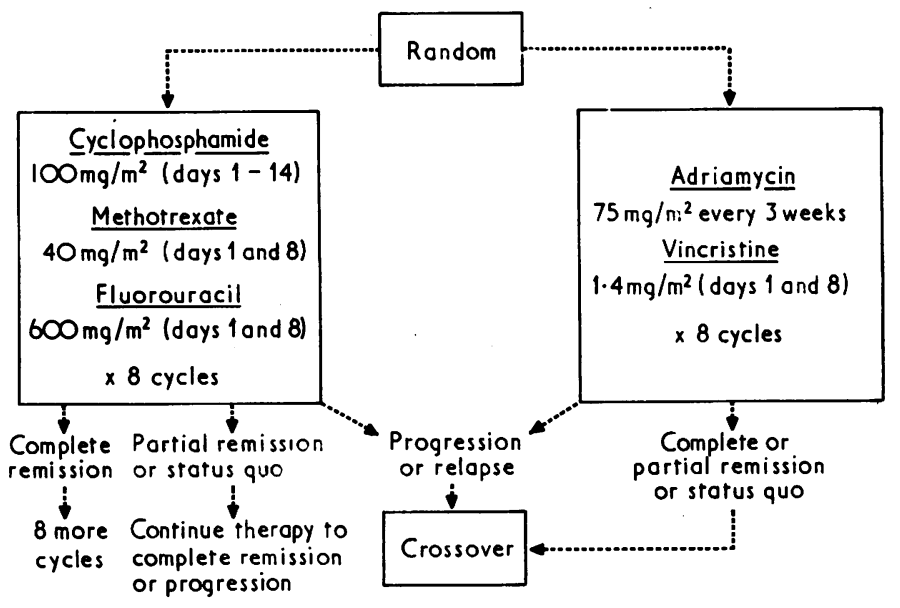

FIG 1-Outline of CMF and AV treatments.

In patients over 60 years or with widespread osseous metastases an initial dose reduction per $\mathrm{m}^{2}$ of body surface was used for adriamycin $(60 \mathrm{mg})$, methotrexate $(30 \mathrm{mg})$, and fluorouracil $(400 \mathrm{mg})$. During treatment a reduced dose schedule was also used, especially when there was myelosuppression. When leucocyte counts were 2.5$3.999 \times 10^{9} / 1\left(2500-3999 / \mathrm{mm}^{3}\right)$ or platelet counts $75-129 \times 10^{2} / 1$ (75 $000-129000 / \mathrm{mm}^{3}$ ), or both (grade 1 toxicity), half of the calculated dose of each drug was given. For lower levels (grade 2 toxicity) treatment was discontinued until at least grade 1 toxicity was reached. Patients who started on adriamycin plus vincrinstine (AV) and showed response or stabilisation after eight cycles were crossed over to cyclophosphamide, methotrexate, and fluorouracil (CMF) to avoid the risk of adriamycin-induced cardiomyopathy.

From March 1973 to June 1974110 patients were admitted to the study. The patients selected were in fairly good condition with measurable disease, an adequate peripheral blood count, and normal electrocardiogram, blood urea nitrogen, serum creatinine, and, with two exceptions, liver function. No patient had yet received chemotherapy. Both treatment groups were comparable in median age (CMF 52 years, AV 48 years) and the proportion that had received hormone treatment (CMF 43\%, AV 40\%).

The criteria of response to treatment have been reported in detail elsewhere. ${ }^{2}$ Briefly, the following definitions were used: complete remission-all objective and subjective signs of disease had disappeared and all osteolytic metastases had recalcified; partial remission-a reduction of $50 \%$ more in the areas of all measurable tumour had occurred and osteolytic lesions had partially recalcified; objective improvement $-25-50 \%$ decrease in the areas of all measurable lesions had occurred with no change in osseous metastases; progressionoriginal measurements had increased by $25 \%$ or more; relapsenew lesions had appeared or measurable tumour had increased by $50 \%$ over the initial size after a period of initial response or no change.

Survival was measured from the start of combination chemotherapy and calculated by the use of standard-life table methods. The differences were tested using the Mantel modification of the Wilcoxon test. $^{8}$ Baseline and follow-up studies included roentgenograms of chest, skull, spine, and pelvis, liver scans, measurements of blood urea nitrogen, serum creatinine, liver function, serum calcium and phosphorus, and a differential blood count before each drug course. All patients were treated as outpatients.

\section{Results}

Five patients were not considered evaluable either because they were lost to follow-up ( 1 case) or because they died early of progressive disease after the first cycle of treatment (4 cases). Therefore 105 patients (CMF 53, AV 52) were suitable for comparative analysis.

Table I shows the response after primary treatment for patients evaluated at eight and more than eight cycles. The analysis failed to show a significant difference in any type of response between the two treatments. In the CMF group more than eight cycles produced a slight increase in the incidence of partial remissions, while in the AV group, further treatment with CMF after the initial eight cycles doubled the number of complete responders. Complete remissions were observed in both groups with different patterns of distribution. Complete recalcification of osteolytic metastases occurred in five patients (CMF 2, AV 3). The median time to achieve complete remission was also similar in both groups (CMF 6 months (range 3-9); AV 5.5 months (range 3-11)).

The response (complete and partial remissions) related to site and dominant lesion is shown in table II. Both combinations were particularly effective in soft tissue disease. No statistically significant difference was observed between $\mathrm{CMF}$ and $\mathrm{AV}$ in the percentage of patients

TABLE I-Comparative response at eight and more than eight cycles

\begin{tabular}{|c|c|c|c|c|c|c|c|c|}
\hline & \multicolumn{4}{|c|}{ CMF (53 Cases) } & \multicolumn{4}{|c|}{ AV (52 Cases) } \\
\hline & \multicolumn{2}{|c|}{8 Cycles } & \multicolumn{2}{|c|}{$>8$ Cycles } & \multicolumn{2}{|c|}{8 Cycles } & \multicolumn{2}{|c|}{$+\mathrm{CMF}$} \\
\hline & No & $\%$ & No & $\%$ & No & $\%$ & No & $\%$ \\
\hline $\begin{array}{l}\text { Objective improvement } \\
\text { Partial remission } \\
\text { Complete remission } \\
\text { Complete plus partial }\end{array}$ & $\begin{array}{r}11 \\
20 \\
5\end{array}$ & $\begin{array}{c}21 \\
38 \\
9.5\end{array}$ & $\begin{array}{r}7 \\
24 \\
6\end{array}$ & $\begin{array}{l}13 \\
45 \\
11\end{array}$ & $\begin{array}{r}6 \\
23 \\
4\end{array}$ & $\begin{array}{c}11 \cdot 5 \\
44 \\
8\end{array}$ & $\begin{array}{r}6 \\
19 \\
8\end{array}$ & $\begin{array}{l}11.5 \\
36.5 \\
15.3\end{array}$ \\
\hline $\begin{array}{l}\text { remission } \ldots \\
\text { Total No responding. }\end{array}$ & $\begin{array}{l}25 \\
36\end{array}$ & $\begin{array}{l}47 \\
68\end{array}$ & $\begin{array}{l}30 \\
37\end{array}$ & $\begin{array}{l}57 \\
70\end{array}$ & $\begin{array}{l}27 \\
33\end{array}$ & $\begin{array}{l}52 \\
63\end{array}$ & $\begin{array}{l}27 \\
33\end{array}$ & $\begin{array}{l}52 \\
63\end{array}$ \\
\hline
\end{tabular}

TABLE II-Complete plus partial responses related to sites of metastases and sites of dominant lesions

\begin{tabular}{|c|c|c|c|c|c|c|c|}
\hline & & & & \multicolumn{2}{|c|}{$\begin{array}{c}\text { CMF } \\
\text { (53 Cases) }\end{array}$} & \multicolumn{2}{|c|}{$\underset{(52 \text { Cases })}{A V}$} \\
\hline & & & & No & $\%$ & No & $\%$ \\
\hline $\begin{array}{c}\text { Metastatic site: } \\
\text { Breast } \ldots \\
\text { Skin } \ldots \\
\text { Nodes } . . \\
\text { Bone } \quad \ldots \\
\text { Lung } \ldots \\
\text { Liver } \ldots \\
\text { Serous cavities }\end{array}$ & $\begin{array}{l}\because \\
\because \\
\ddot{z} \\
\therefore\end{array}$ & $\begin{array}{l}\ldots \\
\because \\
\cdots \\
\cdots \\
\cdots\end{array}$ & $\begin{array}{l}. \\
\because \\
\because \\
\cdots \\
\cdots\end{array}$ & $\begin{array}{c}17 / 30 \\
15 / 27 \\
19 / 27 \\
5 / 19 \\
7 / 14 \\
3 / 9 \\
6 / 6\end{array}$ & $\left.\begin{array}{r}57 \\
55 \\
70 \\
26 \\
50 \\
33 \\
100\end{array}\right\} 61$ & $\begin{array}{c}17 / 27 \\
18 / 29 \\
14 / 19 \\
4 / 15 \\
4 / 14 \\
1 / 4 \\
5 / 9\end{array}$ & $\left.\begin{array}{l}63 \\
62 \\
74 \\
27 \\
28 \\
25 \\
55\end{array}\right\} 65$ \\
\hline $\begin{array}{l}\text { Dominant site: } \\
\text { Soft tissue } \\
\text { Bone } . . \\
\text { Viscera }\end{array}$ & $\begin{array}{l}. \\
\cdots\end{array}$ & $\begin{array}{l}\cdots \\
\cdots\end{array}$ & $\begin{array}{l}\because \\
\because .\end{array}$ & $\begin{array}{r}17 / 28 \\
6 / 10 \\
7 / 15\end{array}$ & $\begin{array}{l}61 \\
60 \\
47\end{array}$ & $\begin{array}{c}17 / 28 \\
4 / 8 \\
6 / 16\end{array}$ & $\begin{array}{l}61 \\
50 \\
38\end{array}$ \\
\hline
\end{tabular}


in whom soft tissue and bone responded. In contrast, CMF produced a higher response rate than $\mathrm{AV}$ for lung, liver, and serous disease $(55 \% v 37 \%)$. This difference was also evident when the response was analysed in terms of the dominant site of the disease. Menopausal status and free interval did not significantly affect the response rate in both groups.

The median length of complete plus partial response was not significantly different between the treatment groups (fig 2). In complete responders who relapsed the disease did not always recur in the original sites (in 1 out of 3 in CMF group and 3 out of 7 in AV group). Furthermore, in five patients (CMF 2, AV 3) new disease occurred in the central nervous system.

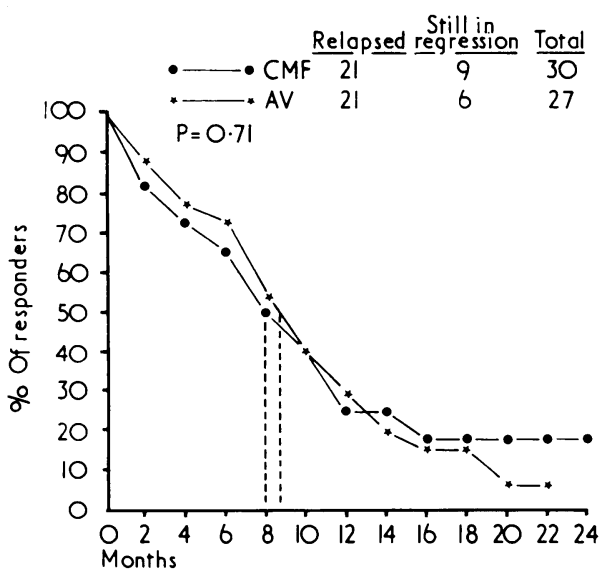

FIG 2-Length of remission duration according to treatment group. Vertical lines indicate median values.

The actuarial analysis of survival for both series is presented in fig 3. In both treatment groups an identical pattern was observed. There was no significant difference between $\mathrm{CMF}$ and $\mathrm{AV}$ in the median survival time (17.5 months $v 22.5$ months). In both groups the survival curve for complete plus partial responders was significantly better than that for patients with no response or only objective improvement (figs 4 and 5).

After crossover for progressive disease eight out of $23(35 \%)$ evaluable patients responded to AV as secondary treatment (partial response 5 , improvement 3 ) for a median duration of 4.5 months. This finding was observed in 3 out of $15(20 \%)$ patients given CMF (all with partial remission) for a median of 7.5 months.

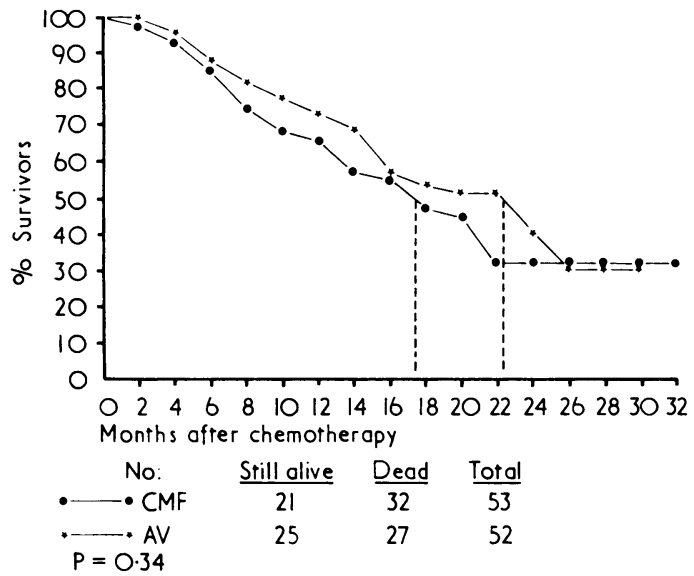

FIG 3-Survival according to treatment group. Vertical lines indicate median values.

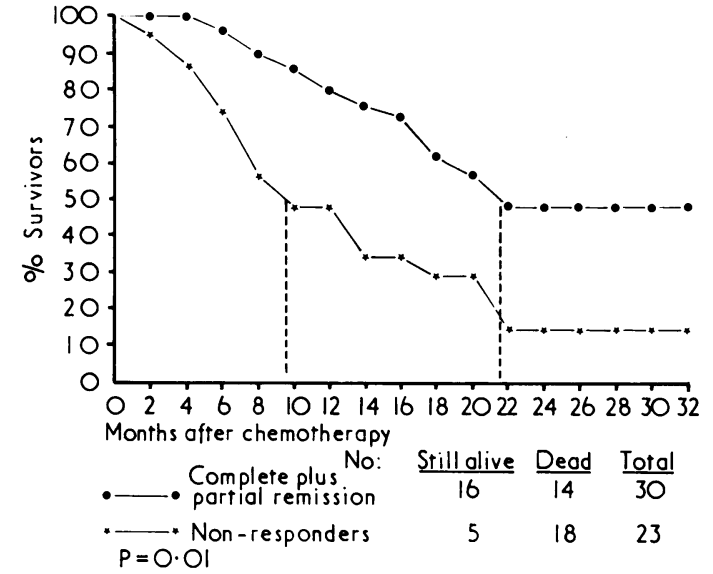

FIG 4-CMF group: survival according to response. Vertical lines indicate median values.

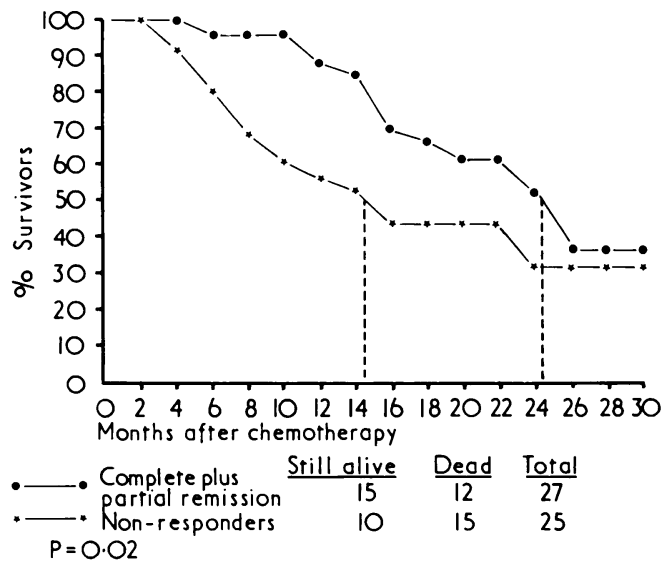

FIG 5-AV group: survival according to response. Vertical lines indicate median values.

Toxic reactions are listed in table III. Bone marrow suppression represented the dose-limiting factor in both treatments. Severe haemosuppresion (leucocytes $<2.5 \times 10^{9} / 1\left(2500 / \mathrm{mm}^{3}\right)$ or platelets $<75 \times 10^{9} / 1\left(75000 / \mathrm{mm}^{3}\right)$, or both) occurred in less than $10 \%$ of patients, with the exception of leucopenia on AV treatment $(22 \%)$. Hair loss to complete alopecia and neurotoxicity were prominent findings in those on AV treatment. Stomatitis was usually moderate and almost never needed a reduction in dose. In contrast, pronounced alopecia was rare with CMF, and chemical cystitis occurred in about a quarter of patients. One patient died from drug-related causes after eight full cycles of adriamycin (total $600 \mathrm{mg} / \mathrm{m}^{2}$ ) without showing bone marrow depression. About one month after crossover to CMF she died at home of irreversible congestive heart failure.

The mean percentages of the optimum doses $\left(\right.$ per $\left.\mathrm{m}^{2}\right)$ that were actually given were: cyclophosphamide $78 \%$, methotrexate $84 \%$, fluorouracil $84 \%$, adriamycin $86 \%$, and vincristine $78 \%$. That such high proportions of the optimum dose of each drug were given indicated that both regimens were fairly well tolerated.

\section{Discussion}

Despite differences in the incidence of complete plus partial remissions, the duration of response and the median survival seems to be remarkably similar on practically all combined

TABLE III-Percentage of each treatment group with various toxic reactions

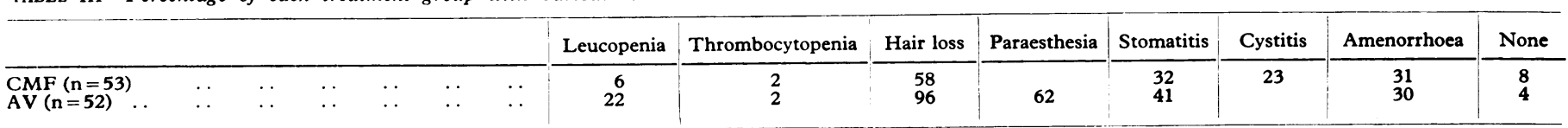


intermittent drug regimens for advanced breast cancer. ${ }^{1}{ }^{3}$ Furthermore, once the disease becomes unresponsive to multiple drug combinations it is difficult to give further effective chemotherapy to patients who have been exposed to major cytotoxic agents. The development of independent non-cross-resistant combinations is therefore of practical importance.

Our two different combinations proved equally effective in terms of response rate, duration of remission, and median survival. On both treatments the median survival of complete plus partial responders was significantly longer than that of patients with no response or only objective improvement. Considering both duration of response and median survival our results are comparable to those reported with similar or different combinations.

Our findings indicate that in patients with advanced breast cancer previously untreated with chemotherapy a two-drug combination (AV) is as effective as a three-drug regimen (CMF). The results of this and other prospective studies ${ }^{6}{ }^{7}$ raise the question of how many drugs and which ones are needed to induce a satisfactory remission rate. The observation that complete plus partial remissions obtained with AV were maintained and even improved by CMF indicates that the duration of response was prolonged by a non-cross-resistant combination when primary treatment included adriamycin. It is well known that treatment with adriamycin cannot be safely continued once the total dose exceeds $500-600 \mathrm{mg} / \mathrm{m}^{2}$. Although secondary treatment is usually less effective than primary treatment, we can conclude that no cross resistance exists between CMF and AV.

Both treatments produced partial and even complete radiographic healing of osteolytic bone metastases in about a quarter of patients. This result, which has previously been noted only after endocrine treatment, can now be obtained also by prolonged combination regimens. Owing to the limited number of patients the question of the influence of free interval and menopausal status on the response rate cannot be answered fully. Available data suggest, however, that these pretreatment variables did not significantly influence the incidence of complete plus partial response.
Considering our results within the framework of present combined drug regimens for advanced breast cancer, we conclude that although combination chemotherapy has improved the control of disease compared with single-agent treatment, no multiple drug regimen has so far proved to be definitely better than another in terms of duration of response and survival. From this point of view, combination chemotherapy alone seems to have almost reached a plateau in its capacity to control disseminated breast cancer. While the initial promising results of treatment with synergistic combinations ${ }^{4}{ }^{5}$ should be confirmed by long-term analysis, new possibilities to improve the duration of response may lie in appropriate sequential combinations or in combining multiple-drug regimens with immunotherapy ${ }^{9}$ or endocrine therapy. The extensive tumour burden present in most patients with clinically disseminated breast cancer presents an unfavourable kinetic condition to available chemotherapeutic combinations. Nevertheless, the therapeutic approaches mentioned above are more likely to improve the duration of remission and survival of responders than to increase the response rate.

This work was supported in part by contract NO 1-CM-33714 with the National Cancer Institute. National Institutes of Health, Division of Cancer Treatment, USA.

Requests for reprints should be addressed to Dr G Bonadonna, Istituto Nazionale Tumori, Via Venezian 1, Milan 20133, Italy.

\section{References}

${ }^{1}$ Broder, L E, and Tormey, D C, Cancer Treatment Reviews, 1974, 1, 183. 2 De Lena, M, et al, Cancer, 1975, 35, 1108.

${ }^{3}$ Canellos, G P, et al, Annals of Internal Medicine, in press.

4 Jones, S E, Durie, B G M, and Salmon, S E, Cancer, 1975, 36, 90.

${ }_{5}$ Blumenschein, G R, et al, Proceedings of the American Association for Cancer Research, 1974, 15, 193.

${ }^{6}$ Ahman, D L, et al, Cancer Chemotherapy Reports, 1974, 58, 877.

7 Gottlieb, J A, et al, Adriamycin Review, EORTC International Symposium. European Press Medikon, 1975.

${ }^{8}$ Mantel, M, Biometrics, 1967, 23, 65.

9 Cardenas, J O, et al, Cancer Immunology and Immunotherapy, 1976, in press.

\title{
Rheumatoid arthritis and ankylosing spondylitis occurring together
}

\author{
GEORGES H FALLET, MICHAEL MASON, HEDLEY BERRY, ALASTAIR G MOWAT, \\ IRADJ BOUSSINA, JEAN-CHARLES GERSTER
}

British Medical fournal, 1976, 1, 804-807

\section{Summary}

Rheumatoid arthritis and ankylosing spondylitis are often difficult to differentiate, though it is important to

\section{Division de Rhumatologie, Hôpital Beau-Séjour, Geneva}

G H FALLET, MD, professor

I BOUSSINA, $\mathrm{MD}, 1$ st senior registrar

J-C GERSTER, MD, 2nd senior registrar

Department of Rheumatology, The London Hospital, London E1 1BB

MICHAEL MASON, DM, FRCP, consultant physician

H BERRY, DM, MRCP, senior registrar (now consultant physician, Department of Rheumatology, King's College Hospital, London SE5)

Department of Rheumatology, Nuffield Orthopaedic Centre, Headington, Oxford

A G MOWAT, MB, FRCP, consultant rheumatologist do so as the natural history and treatment of the two conditions differ. Nine patients have recently been seen, each of whom fulfilled the criteria for both rheumatoid arthritis and ankylosing spondylitis. In eight of the nine patients the histocompatibility antigen HLA-27 was present. A possible explanation of these cases is that one of the diseases occurred by chance in patients already suffering from the other, but this is extremely unlikely. If a chance association is not the correct explanation the basic concepts defining rheumatoid arthritis and ankylosing spondylitis must be reconsidered.

\section{Introduction}

The distinction between rheumatoid arthritis and ankylosing spondylitis has relied considerably on the results of the RoseWaaler and latex tests, which detect the IgM component of rheumatoid factor. Despite their well-recognised defects of false-positives and occasional false-negatives, these remain 